\title{
3 Research Square \\ Fried Carbohydrate-rich Food as a Potential Source of Malondialdehyde and Acrylamide: a Consumption Pattern to Evaluate the Risk of Cancer in Population
}

\section{Sheno Karimi}

Kermanshah University of Medical Sciences

\section{Farjam Goudarzi}

Kermanshah University of Medical Sciences

Mojtaba Pourmehdi

Kermanshah University of Medical Sciences

\section{Fatemeh Heydarpour}

Kermanshah University of Medical Sciences

\section{Behzad Mahaki}

Kermanshah University of Medical Sciences

\section{Seyed Mostafa Nachvak ( $\nabla$ smnachvak@hotmail.com )}

phd in nutrition, faculty of Nutrition Department, Schoole of Nutrition and food Technology,Kermanshah university of Medical Science, Kermanshah, Iran

\section{Research}

Keywords: Fried food, Acrylamide, Malondialdehyde, ILCR, Consumption pattern, Cancer

Posted Date: February 15th, 2021

DOI: https://doi.org/10.21203/rs.3.rs-191271/v1

License: (c) (1) This work is licensed under a Creative Commons Attribution 4.0 International License. Read Full License 


\section{Abstract}

Background: Tah-Dig (A food rich in carbohydrate that is highly fried) is one of the most popular and tasty Persian food. However, toxic compounds such as malondialdehyde (MDA) and acrylamide are vastly formed in Tah-Dig. The purpose of this study was to investigate the cooking and consumption pattern, intake frequency, acrylamide and malondialdehyde content of Tah-Dig in Iranian population.

Methods. The content of acrylamide consumption in population was measured by LC-MS/MS and the MDA in cooking oils used to prepare Tah-Dig was also quantified. Data mining was used to investigate the relationship between the influential factors and Incremental Lifetime Cancer Risk (ILCR).

Results. The most consumed type of Tah-Dig among Iranians is Tah-Dig of bread. The highest amount of acrylamide consumed by population was found in potatoes Tah-Dig. The highest MDA content was detected in canola frying oil.

Conclusions. Considering data mining analysis, it was shown that the most influential factors related to ILCR are a cumulative factors of Tah-Dig color, portion size, Tah-Dig type, oil type, and the level of education.

\section{Introduction}

The food contains macronutrients, micronutrients, minerals, and vitamins that dietary habits such as methods of cooking can affect these nutrients. Cuisines refer to the traditional methods of cooking and consumption common in each country and are one of the factors that can be beneficial or harmful to health [1]. Rice is the chief staple food of the Iranian diet [2]. Tah-Dig is typically made with rice, which is one of the most popular and delicious cuisines of the Iranian people [3]. Tah-Dig is a thin crunchy and slightly browned rice or bread or potato which is formed in the process of cooking rice at the bottom of the pot $($ Tah $=$ Bottom + Dig = Pot). This discoloration is due to the Maillard reaction, which is a nonenzymatic reaction between free amines and carbonyl groups of carbohydrates exposed to high heat by cooking methods (fried, cooked, and roasted foods) [4]. Tah-Dig can be included potatoes, rice, bread, lettuce, sesame, baguettes, etc. However, Tah-Dig of bread, potato and rice are more common than the others.

In the cooking process, acrylamide is one of the by-products produced by high-temperature cooking of the foods. Acrylamide is a water-soluble white crystal that is mostly used in industry as a material for dam construction, tunneling, paper industry, gel electrophoresis, and water treatment [5]. According to a report by the International Agency for Research on Cancer in 1994, it has been identified as a potential carcinogen for humans [6]. Further experiments [7] confirmed the possibility of carcinogenicity in humans [8]. Before 2002, acrylamide was thought to accumulate in the human body, mostly through occupational exposure to cigarette smoke, and rarely through drinking water and cosmetics, but in a preliminary study in Sweden on food Rich in starch exposed to high temperatures, high levels of acrylamide were observed. Further studies in other sources have shown that acrylamide is found in fats [9] and proteins, but is most 
commonly found in carbohydrate-rich foods $(150-4000 \mu \mathrm{g} / \mathrm{g})$. Other research has been done to measure acrylamide level in various foods. Various methods such as HPLC [10], LC-MS/MS, and GC /MS can be used to measure acrylamide levels, but LC-MS/MS method is one of the most widely applied techniques due to its high sensitivity and accuracy [11].

Other ingredients used in cooking rice are solid and liquid oils. Oxidation, resulting from high temperature, is a notable feature of oils. Long-time exposure of oil to a high temperature for producing a crunchy layer of rice at the bottom of the pot intensifies the oil oxidation process leading to MDA production, a carcinogenesis compound that is bound to DNA and changing the signaling pathways [12]. The degree of lipid oxidation depends on the percentage of saturated and unsaturated hydrocarbons[13]. MDA is the best indicator for detecting lipid oxidation [14].

Genetic predisposition is responsible for only $5-10 \%$ of cancers, whereas $90-95 \%$ of cancers have their roots in the lifestyle.30-35\% of cancer incidence may be related to negative dietary habits [15]. Cancer is the second leading cause of death in the United States [16] and the third leading cause of death in Iran [17]. Due to the high economic, psychological, and social burden of cancer treatment, in recent years, significant attention has been paid to identifying patterns for cancer prediction and prevention. Cancerrelated predictive indicators include incremental lifetime cancer risk index (ILCR) [18] as well as predictive data mining equations that can predict cancer-related patterns by considering the existing risk factors [19].

As cooked rice is one of the most popular dishes in Iran and Tah-Dig, is an integral part of this popular food, the following questions arise; How much is the consumption of Tah-Dig among Iranian people? According to the ILCR index and data mining equations, is there any relationship between Tah-Dig consumption and risk of cancer incidence among children and adults?

\section{Materials And Methods}

\subsection{Questionnaire}

To investigate the consumption and cooking patterns of Tah-Dig among the community, a questionnaire containing 34 questions was designed taking into account the cooking methods, consumables, and also the types of interests of the people. For content validity, $\mathrm{CVR}=$ Content validity ratio and $\mathrm{CVI}=$ Content validity Index were calculated. Five nutritionists, three food industry specialists, a statistician, and an epidemiologist familiar with Iranian cuisines were consulted. Questions that did not meet the quorum of $C V R=0.062$ and $C V I=0.79$, and had less than $5 \%$ response were eliminated. To evaluate the reliability, Test-Retest was used in a pilot population equivalent to $10 \%$ of the prototype volume at 15 -day intervals. Subjects with a special diet were excluded from this study. The final questionnaire consisting of 25 questions was prepared. KUPPUSWAMY's SES scale was used for socio-economic ranking with three criteria of the job, education, and monthly income. Individuals' monthly income was measured indirectly based on the level of the urban area. Three levels of SES were considered high, medium, and low. 


\subsection{Determine the sample and size of the population}

The cross-sectional, descriptive-analytical study was conducted on the population of Kermanshah (Western Iran) from 2019 to 2020. To identify possible differences in cooking and consumption methods among Iranian population, questionnaires were randomly completed in four other cities besides Kermanshah. The sample size considering the confidence level of $P=0.095$ which is proportional to the consumption of Tah-Dig in the community and due to the lack of similar studies, 600 people for the city of Kermanshah (with eight urban areas) as the study center and the cities of Sari (north), Isfahan (center), Bandar Abbas (south) and Hamedan (west) a total of 150 people were identified. The sample size was proportional to the total population of that area. 48 and 270 Tah-Dig in pieces were required to measure the levels of the acrylamide and malondialdehyde content, respectively.

\subsection{Sample collection and categorization}

Participants were asked to provide us Tah-Dig in one piece cooked in the same way as usual in the time allotted for collecting the sample, and because of the stability of acrylamide and oxidized fatty acids we stored them in a $-20^{\circ} \mathrm{C}$ freezer until delivery. A total of 343 samples were collected. Due to the nonuniform cooking conditions such as temperature, time, and type of oil in households, unlike laboratory conditions, the color was considered as a criterion, and samples with similar colors were placed in a group due to having the same amount of acrylamide. The samples were compared using Adobe Photoshop CC20.0.1 (Figure 1), and the samples with the same RAL and mean RBG \pm 15 were grouped (Table 1). The classification was based on the spectrum of colors in gold, light brown, and dark brown for each type of Tah-Dig. Four control samples of high-consumption flat breads in Iran (Lavash, Taftoon) used in baking Tah-Digs were also prepared. Oils were classified based on the three main groups (solid, liquid, and combined) and nine subgroups (Kermanshahi oil, vegetable solid, canola cooking oil, sunflower cooking oil, oil Sunflower frying and combination of Kermanshahi oil with other oils). Kermanshahi oil is prepared by animal butter especially sheep butter, with a pleasant taste and smell. 
Table 1

Classification of Tah-Dig samples based on color analysis (RBG).

\begin{tabular}{|lllll|}
\hline Type of Tah-Dig & Color gradẽ & Red & Green & Blue \\
\hline Rice & R1 & $194.2 \pm 12.93$ & $175.4 \pm 12.9$ & $97.36 \pm 16.36$ \\
\hline & R2 & $188.36 \pm 14.5$ & $166.45 \pm 8.9$ & $60.36 \pm 15.22$ \\
\hline R3 & $147.20 \pm 35.7$ & $109.00 \pm 42.6$ & $34.00 \pm 12.36$ \\
\hline Bread & B1 & $215.40 \pm 15.23$ & $187.70 \pm 27.166$ & $82.90 \pm 24.97$ \\
\hline & B2 & $182.10 \pm 12.81$ & $134.00 \pm 16.06$ & $14.00 \pm 7.3$ \\
\hline Potato & B3 & $163.00 \pm 28.26$ & $109.00 \pm 32.42$ & $15.20 \pm 7.16$ \\
\hline & P1 & $232.70 \pm 3.16$ & $196.00 \pm 17.98$ & $80.80 \pm 26.39$ \\
\hline & P2 & $204.90 \pm 5.01$ & $176.10 \pm 13.81$ & $48.5 \pm 15.50$ \\
\hline & P3 & $208.50 \pm 8.700$ & $110.90 \pm 15.98$ & $6.900 \pm 3.695$ \\
\hline
\end{tabular}

* The numbers represent the pixels counted by color and are reported as Mean \pm SD.

- The samples had a significant difference $(P<0.0001)$ within the group at the color level, which represents three separate color spectrums.

- $\mathrm{R}=$ rice, $\mathrm{B}=$ bread, $\mathrm{P}=$ potato. Grades 1,2 , and 3 indicate an increase in color.

\subsection{Statistical analyses}

In the present study, central tendency indicators such as mean and standard deviation or percentage for continuous and classified variables were used to describe the data. Due to the method and nature of the research, analysis of variance (ANOVA) was used to compare continuous demographic variables and the Chi-square test was used for variables classified into different amounts of consumption. Data were analyzed using SPSS 24.0 software package. The data were collected precisely according to hypothesis and the methods were planned in-line with major purpose of the study.

\subsection{Standard preparation for acrylamide}

Stock solutions of acrylamide and acrylamide-d3 (Sigma-Aldrich, USA) were diluted to $1 \mathrm{mg} / \mathrm{ml}$ in deionized water. A standard diluted solution of $10 \mu \mathrm{g} / \mathrm{ml}$ in deionized water $(18 \Omega)$ was then prepared. Tah-Dig samples were evaluated within the dynamic standard range of acrylamide $20-3000 \mathrm{ng} / \mathrm{ml}$. To prevent light exposure, all standard solutions were placed in a volumetric flask and kept at $4{ }^{\circ} \mathrm{C}$ for 4 hours. Carrez I solution was prepared by dissolving $1.5 \mathrm{~g}$ of potassium hexacyanoferrate in $10 \mathrm{ml}$ of water, and Carrez II solution was prepared by dissolving $3 \mathrm{~g}$ of zinc sulfate in $10 \mathrm{ml}$ of water to make diluted standard solutions. 


\subsection{Extraction of acrylamide}

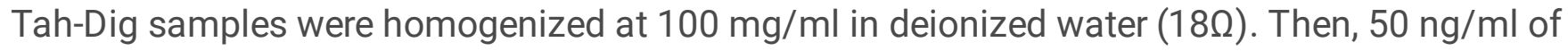
acrylamide-d3 as internal standard (Sigma-Aldrich, Germany) was added to the samples and incubated for $20 \mathrm{~min}$ at $4{ }^{\circ} \mathrm{C}$ in the dark to allow for equilibration. It was then centrifuged at $6000 \times \mathrm{g}$ for 20 minutes. One milliliter from the aqueous phase of the supernatant was removed and $650 \mu \mathrm{l}$ of supernatant was purified by Oasis HLB 6 cc Vac Cartridge, 200 mg Sorbent (Waters Corp, USA). The samples were then stored at $-20{ }^{\circ} \mathrm{C}$ until analysis.

\subsection{LC-MS/MS analysis}

Samples were analyzed by LC-Tandem Mass (Quattro Micro API micro mass Waters 2695, USA) [1]. EC HPLC column (analytical), NUCLEODUR C18 Gravity, $5 \mu \mathrm{m}, 250 \times 2 \mathrm{~mm}$ (MACHERY-NAGEL, GERMANY) was used for chromatography with isocratic conditions: Methanol: Formic acid (94: 5: 1) was used at a flow rate of $1 \mathrm{ml} / \mathrm{min}$. The LC-MS/MS parameters were as follows: Source temperature $500^{\circ} \mathrm{C}$; Separation Gas Collision (CAD) 5; Nebulizer gas (GS1) and auxiliary gas (GS2) below 40 psi and 50 psi, respectively, and curtain gas (CUR) below 10 psi. Declining potential (DP) and collision energy (CE). Finally, a general scan was performed to determine the standard $\mathrm{m} / \mathrm{z}$ ratio of acrylamide and acrylamided3 to select the most appropriate mass spectrum [20]. Dynamic range was considered 15-1000 ng/ml and LOQ was considered $15 \mathrm{ng} / \mathrm{gram}$ of Tah-dig that the device could detect.

\subsection{MDA Extraction and Evaluation}

Tah-Digs samples were homogenized at $100 \mathrm{mg} / \mathrm{ml}$ in $50 \%$ acetic acid. It was then centrifuged at $6000 \times$ $\mathrm{g}$ for 20 minutes and the supernatant was stored at $-80^{\circ} \mathrm{C}$ until the time of the experiment.

The commercial lipid peroxidation kit (Kiazist Life Sciences, IRAN) was used to measure MDA. $200 \mu$ l of samples, standards, and blanks were poured into the microtubes, then $600 \mu \mathrm{l}$ of TBA solution in the kit was added and the lids of the microtubes were tightly sealed with parafilm to prevent evaporation. It was then incubated for one hour at $95^{\circ} \mathrm{C}$. After incubation, the samples were cooled to room temperature and $200 \mu \mathrm{l}$ of samples were transferred into a 96-well plate of the kit and their absorption was read at $560 \mathrm{~nm}$ by plate reader.

\subsection{Measurement of triglycerides}

Due to the difference in the amount of oil consumed for making Tah-Digs, MDA samples were evaluated based on grams of oil consumed. The triglyceride content of the samples was measured by the commercial kit (Pars Azmoon, Iran) to normalize the MDA values. Triglyceride in the samples was evaluated by the glycerol phosphate dehydrogenase (GPO) method. Homogenized samples were exposed to Working Solution for 10 minutes at room temperature to measure MDA. Afterwards, the absorption of TG at $500 \mathrm{~nm}$ was measured by a plate reader (BioTek Instruments' USA) and the TG value of the samples was calculated based on the standard absorption diagram. 


\subsection{Risk assessment}

The incremental lifetime cancer risk equation (ILCR) was used to assess the risk of Tah-Dig consumption:

$\mathrm{ILCR}=\mathrm{CDI} \times \mathrm{CSF}$

The ILCR is an estimate of chroninc daily intake (CDI) of the chronic daily intake of acrylamide (mg / $\mathrm{kg} /$ day), the values of which are based on the average intake based on questionnaire data and measurement of LC-MS/MS acrylamide. The equation is shown below. CSF equivalent to cancer slope factor -1 (mg / kg-day), which was considered equal to 0.5 based on 2009 Oehha [20].

$C D I=\frac{C * I R i * E F i * E D i}{B W * A T}$

The CDI is derived from the above-mentioned equation in which C: the amount of acrylamide per gram Tah-Dig, IRi: the amount of Tah-Dig received based on the defined serving (the amount of Tah-Dig consumed per person according to the usual cooking utensils on the market (sizes $18 \mathrm{~cm}, 24 \mathrm{~cm}, 26 \mathrm{~cm}$ and above, with an estimated amount of 300, 200 and $100 \mathrm{~g}$ of Tah-Dig). Then, the amount of Tah-Dig (gram) was divided by the number of family members to get the consumption (per person (g) / 365), BW: Individual weights $(45,65$, and $70 \mathrm{~kg})$, which were defined for people aged 18,20 years and those over 70 years, respectively. EDi: years of consumption (due to not consuming Tah-Dig in childhood, the age minus four was considered), and EFi: exposure time per year (repeated per week $\times 56$ ).

\subsection{Data mining analysis of Tah-Dig cooking patterns related to cancer risk}

Data mining analysis is a process to extract implicit patterns based on the factors leading to the prediction of an event. This study aimed at developing a predictive model to categorize the participants into two groups: those at high risk of cancer and those with no known risks based on the answers to the questions in the questionnaire and the effect they had on ILCR; therefore, the decision tree algorithm was used [21]. According to this classification, items such as high flame temperature, MUFA, and PUFA lipids, and fried Tah-Dig texture were found to be associated with high levels of acrylamide, while low flame temperature, SFA oils, and soft tissue of Tah-Dig were found to be associated with low levels of acrylamide. Other questionnaire responses were also included in the program. The ILCR index was defined as a dependent variable and other questionnaire questions were defined as an independent variable. Finally, the accuracy and performance of the model for use in similar conditions were evaluated.

\section{Results}

\subsection{Consumption pattern evaluation}

Figure 2 provides information on age, education, occupation, number of family members, and SES. About $40 \%$ of households consumed rice seven times per week (Table $A$ as supplementary for details). $82.5 \%$ of 
households always had rice with Tah-Dig. Households consuming different types of bread, rice, and potato Tah-Digs were $41.4 \%, 37.2 \%$, and $21.4 \%$, respectively (Figure $2 \mathrm{~A}$ ). $89.2 \%$ of the participants tended to eat fried Tah-Dig. About half of the families reported cooking time of 15-30 minutes (Figure 1B) and $34 \%$ of the participants chose the option of using a medium flame. $12.3 \%, 62 \%$, and $25.7 \%$ of households consumed a quarter, half, and the total amount of rice Tah-Dig, respectively. Participants showed their tendency towards cooking and eating Tah-Digs as follows: golden brown (16\%), light brown (57.8\%) and dark brown (26.2\%), respectively. The most requested Tah-Dig among households was golden-brown layer with potatoes (26\%) and then the bread with dark brown color (19.3\%). $98.2 \%$ of the cases stated that if the color of the bottom of the pot becomes darker, they will use it again. There was no significant difference between SES at all three levels and the type of oil selected by households. Moreover, there was no significant difference between the choice of the type of Tah-Dig consumption and the social level of households.

\subsection{LC-MS/MS of acrylamide}

94.4\% of Tah-Dig samples were in the range of LOQ. It was also seen that samples of Taftoon and Lavash bread were below the LOQ before baking. The overall mean of acrylamide of bread Tah-Dig ( $\mathrm{n}=15$ ) was $124.5 \pm 68.5 \mathrm{ng} / \mathrm{g}, 75$, and $125 \mathrm{ng} / \mathrm{g}$ for golden, light brown, and dark brown, respectively. The acrylamide content of rice Tah-Dig was $576 \pm 136$, which was 128,143 , and $203 \mathrm{ng} / \mathrm{g}$ for golden, light brown, and dark brown, respectively, and for potato Tah-Dig was 1097 \pm 637.1 , which was 499,868 , and $1926 \mathrm{ng} / \mathrm{g}$ for golden, light brown, and dark brown, respectively. The most temperature-dependent changes measured by color were observed in Tah-Digs made with potato, bread and rice (Figure 2D). These results were generalized to all respondents based on the colors of Tah-Dig [22].

There was also a significant difference between the acrylamide content of Tah-Dig samples of potato, rice, and bread in three different colors of golden, light brown, and dark brown and in groups (Intragroup assay) $(P<0.005)$ (Figure 3$)$. There was a statistically significant difference between potato and flatbread Tah-Digs in the acrylamide levels $(P<0.05)$. Moreover, a statistically significant difference was observed in the acrylamide levels between rice and potato Tah-Digs $(P<0.001)$. In addition, the findings showed a statistically significant difference between bread and rice Tah-Digs $(P<0.001)$.

\subsection{MDA content of samples}

Liquid cooking oil (23.6\%), Kermanshahi oil (16.9\%), and Kermanshahi oil in combination with other oils (15.5\%) were among the most edible oils used in the process of cooking rice (23.6\%) (Table B as supplementary). The highest MDA values measured before cooking were for canola oil at $354 \mathrm{nmol} / \mathrm{g}$ of TG and the lowest was for the combination of solid vegetable oil and frying oil at $29.9 \mathrm{nmol} / \mathrm{g}$ of TG (Figure 4). Measurements were made at 20, 25, 30, 35, 40, 45, 50, 60, and 65 minutes based on the answers given by the participants in the questionnaire. The highest MDA production after cooking was related to canola cooking oil with an average of $1527.89 \mathrm{nmol} / \mathrm{g}$ of TG, and the lowest value was related to the combination of Kermanshahi oil and frying oil with an average of $546.812 \mathrm{nmol} / \mathrm{g}$ of TG. The most changes were related to Kermanshahi oil at $45^{\circ} \mathrm{C}$, canola oil at $30^{\circ} \mathrm{C}$, and sunflower cooking oil at 
$40^{\circ} \mathrm{C}$. There was a significant relationship between the type of oil consumed and the SES of households $(P<0.001)$. The frequency of Kermanshahi oil among SES1, SES2, and SES3 households was $18.8 \%$, $16.7 \%$, and $14.5 \%$, respectively. The most consumed oils for cooking rice in households with SES1, SES2, and SES3 were olive and sesame oil (23.4\%), cooking oil (28.2\%), and solid vegetable oil (25.8\%), respectively. Furthermore, the percentage of combined oils used in SES1, SES2, and SES3 was $19.3 \%$, $12.8 \%$, and $14.5 \%$, respectively.

\subsection{Prediction of cancer risk}

According to the US-EPA definition, ILCR values of $1 \times 10^{-5}$ were in the safe range for cancer and ILCR $\geq$ $1 \times 10^{-5}$ were in the cancer risk range [20]. In this study, it was found that $24.6 \%$ of people have no risk of cancer. $76.4 \%$ of people had a ILCR with the concept of cancer risk. The relationship between ILCR values and type of Tah-Dig was not significant $(P=0.317)$. There was a significant relationship between ILCR and age $(P=0.005)$ in this study.

Moreover, a strong significant relationship was found between the portion size in each household with ILCR values and the risk of cancer $(P<0.001)$. This correlation between flame intensity and cancer risk was statistically positive and significant $(P<0.001)$. However, the relationship between rice cooking time and at-risk ILCR values was not statistically significant and no correlation was found.

The study showed that there is no significant difference between the level of education and the color of Tah-Digs consumed by households. But, the results showed that there was a relationship between the number of family members and cancer risk prediction $(P<0.001)$.

\subsection{Evaluation of data mining of cooking and consumption patterns.}

Tree diagram displays the application of the decision tree algorithm on the collected data (Figure 5). The nodes in the diagram represent the absence of cancer risk with zero code. The risk of cancers was coded as one and predictive patterns were extracted. From 165 patterns, two-thirds of them were associated with cancer risk, and one-third was associated with non-cancer risk.

About two-thirds of the predicted patterns were related to cancer risk and involved 491 of 611 respondents. The color of Tah-Dig is the most important influential factor related to the risk of cancer. Other variables influence the risk of cancer include SES, level of education, number of times per week, type of Tah-dig, type of oil consumed, and portion size (Table 2). The total number of participants $(n=120)$ were associated with no cancer risk patterns. The accuracy of the models for use in similar patterns was $85.15 \%$.

Table 2 Frequency of effective features in predicting ILCR 


\begin{tabular}{lccccccc}
\hline Features & Color Tah- & Oil & Education & SES & Serving & Type of Tah- & Frequency \\
& Dig & Type & & & Number & Dig & (week) \\
\hline ILCR0* & 43 & 31 & 40 & 24 & 13 & 18 \\
(frequency/pattern) & & & & & & & \\
\hline ILCR1** & 119 & 24 & 38 & 91 & 21 & 30 & 80
\end{tabular}

(frequency/pattern)

* No risk of cancer

** With the risk of cancer

\section{Discussion}

One of the most useful tools for assessing the relationship between the amount and manner of eating with a wide range of chronic diseases and types of cancer is to study the dietary patterns of people in the community. The geometry of nutrition is based on the effect of nutritional patterns on a set of conditions on health and disease, not nutrients alone. Foods are a collection of micronutrients, macronutrients, and toxins, but the point to be considered about their impact on health is the reproducibility of patterns in the society.

Acrylamide is a food contaminant formed in thermal processes, which can cause cancer. Tolerable daily intake (TDI) for acrylamide was estimated to be $2.6 \mu \mathrm{g} / \mathrm{kg}$ [23] in previous studies. In the United States and Europe, acrylamide contains about 30\% of calories [24]. However, since the frequency of rice consumption is high in Iran, there is a possibility of receiving higher amounts of acrylamide through TahDig. In this study, tolerable daily intake (TDI) for acrylamide in $60.9 \%$ of participants was higher than expected values just for consumption of rice Tah-Dig.

In this study, the highest levels of acrylamide were found in Tah-Digs with potato, rice, and bread, respectively as previous studies $[3,20]$. Potato Tah-Digs with higher content of asparagine compared to bread and rice resulted in higher production of acrylamide [25]. Moreover, bread fermentation leads to a decrease in the level of asparagine and conversion of reducing sugars to ethanol lowers the $\mathrm{pH}$ values and production of acrylamide [26]. The amount of acrylamide obtained from the present study was higher than laboratory samples, which indicates that domestic cooking methods will produce higher amounts. Contrary to other studies in which FFQ questionnaire was used to estimate the intake level of acrylamide [27], this study designed a specific questionnaire to identify cooking methods, consuming materials, tendency of people even children as high-risk groups and the amount of consumption in the Iranian society. 
Temperature and time are the most important factors in the formation of acrylamide [28]. Most acrylamide is formed in the first 8 to 10 minutes of cooking time [29] and with increasing cooking time, its production decreases [30]. But, it was also observed that the production of acrylamide increases with increasing cooking time [31]. In our study, there was no significant relationship between the cooking time and the amount of acrylamide, but this relationship was significant with the flame intensity, which may indicate that the cooking temperature is a more important parameter. Temperature and time can be in equilibrium, but the color appears to be a far more accurate indicator of acrylamide levels [22]. It can be said that the formation of acrylamide is in equilibrium with a set of cooking factors that ultimately leads to the formation of acrylamide at the level of a particular color.

In this study, there was no significant difference between the parameters that show people's attitudes towards food contamination and the harmfulness of a particular type of Tah-Dig and Tah-Dig oil with the level of education and different classes of SES. High tendency of people towards eating crunchy rice or Tah-Dig may be affected by some properties such as texture, appearance and taste [32].

In the analysis of oils, two main factors were obtained: A) The amount of MDA before cooking and the slope of changes: This measurement was made to find out which oil contains the maximum amount of MDA before cooking and which oil has the most slope of change. MDA production increased with cooking time [33]. The highest amount of MDA and the slope of changes were related to vegetable oils with polyunsaturated chains such as canola and sunflower for cooking. The higher the ratio of PUFA and MUFA fatty acids in oils, the higher the MDA production [34]. It was also found that the lowest amount of MDA before cooking and the slope of changes over a period of time are related to Kermanshahi oil and the combination of Kermanshahi oil with frying oil, which indicates that the higher the SFA in oils, the lower the amount of MDA [35]. B) Combination of Kermanshahi oil with other oils: The composition of Kermanshahi oil is such that it contains short-chain fatty acids (60\%), medium-chain fatty acids (31.4\%), and long-chain fatty acids (4\%) [36]. When combined with other oils, MDA production decreased due to changes in the composition of fatty acids. It was also observed that the composition of Kermanshahi oil reduced the slope point of changes at lower temperatures, which contradicts the fact that the resistance of saturated oils to high temperatures is high. Regarding oils, it was seen that there was no significant difference between Kermanshahi oil consumption and SES. The special flavor of Kermanshahi oil is the most important factor affecting the choice of cooking oil.

In the higher social classes, the use of sesame and olive oils, which contains more PUFA and MUFA acids, leads to a reduction in cardiovascular diseases [37] If the cooking time for the olive oil to be used in cooking becomes short [38]. The maximum slope of MDA production changes in the first 25-30 minutes of cooking. The concern about the consumption of these oils is the lack of knowledge about their cooking time, which is associated with the risk of cancer if high levels of aldehyde metabolites are produced [39]. Rice is regarded as a cooked food in Iranian culture, but Tah-Dig, which is placed in the bottom of the dish and exposed to heat and oil for at least 15-30 minutes, has the characteristics of fried foods that produce high content of MDA in saturated fatty acids. It seems reasonable to use Kermanshahi oil with other oils at the end of cooking time (less than $25 \mathrm{~min}$ ) due to the reduction of MDA. If people consume laboratory 
levels of Tah-Dig samples, a high percentage of people are at risk of cancer [20]. In the present study, it was also seen that three-quarters of people are at risk of cancer by consuming different types of Tah-Dig, with the difference that these findings were obtained from community. A significant relationship observed between cancer risk and the number of family members per household was related to cooking rice in the traditional way. In this method, more heat and longer time are required for cooking rice in large quantity, resulting in high levels of acrylamide. Furthermore, the positive correlation between portion size and ILCR values indicates that portion size as an independent factor of color and type of Tah-Dig can predict cancers.

Predictive models can largely estimate the factors influencing acrylamide intake and the risk of cancer [21]. In this study, a set of factors, including the color of Tah-Dig [29], the type of oil consumed [3], the portion size [40], the type of Tah-Dig consumed [3,20], social class and education have the most power to predict cancer.

\section{Conclusion}

In this study, a large percentage of participants consumed Tah-Dig per week, which led to the formation of high levels of acrylamide and oxidized fatty acids. Data mining revealed that factors such as lifestyle and cooking methods might be involved in increasing risk of cancer. No significant difference was observed between the amount and manner of Tah-Dig consumption at different socio-economic classes. A large percentage of families will be affected by cancer if present cooking patterns and amount of consumption continue and no modification occurs. Nevertheless, it is important to consider that cooking carbohydrates with oils promotes the formation of toxic substances such as acrylamide and malondialdehyde at the bottom of the pot.

\section{Declarations}

\section{Ethics approval and consent to participate}

The study was performed in accordance with Research Ethics Committee of Kermanshah university of medical sciences that is in-line with NIH guidelines (Ethics code: IR.KUMS.REC.1398.207).

\section{Consent for publication}

Not applicable.

\section{Availability of data and materials}

All data generated or analyzed during this study are included in this published article

\section{Competing interests}

The authors declare that they have no competing interests. 
Funding

The authors thank the Vice Chancellor for Research of Kermanshah University of Medical Sciences for allocating a grant (No. 980216) to perform the thesis for the course of the MSc.

Acknowledgements

Not applicable.

Authors' contributions

SK: Conceptualization, Methodology, Writing - Original Draft. FG: Methodology, Supervision, WritingOriginal draft preparation, Resources. MP: Data Curation. FH: Methodology, Formal analysis. BM: Formal analysis. SMN: Reviewing and Editing, Project administration.

\section{References}

1. Perc M: Beauty in artistic expressions through the eyes of networks and physics. Journal of the Royal Society, Interface 2020, 17:20190686-20190686.

2. Baygi F, Qorbani M, Motlagh ME, Shafiee G, Nouri K, Ahadi Z, Mahdavi-Gorab A, Heshmat R, Kelishadi $\mathrm{R}$ : Is frequency of potato and white rice consumption associated with cardiometabolic risk factors in children and adolescents: the CASPIAN-V study. BMC cardiovascular disorders 2020, 20:239-239.

3. Akbari-adergani B, Ahmadi A, Jahedkhanki G, Nodehi RN, Sadighara P: The Comparative Amount of Acrylamide in Tahdig Prepared with the Most Common Edible Liquid and Solid Oils. Current Nutrition \& Food Science 2020, 16:776-780.

4. Rifai L, Saleh FA: A Review on Acrylamide in Food: Occurrence, Toxicity, and Mitigation Strategies. International Journal of Toxicology 2020, 39:93-102.

5. Kumar J, Das S, Teoh SL: Dietary Acrylamide and the Risks of Developing Cancer: Facts to Ponder. Frontiers in nutrition 2018, 5:14-14.

6. Smoke T, Smoking I: IARC monographs on the evaluation of carcinogenic risks to humans. IARC, Lyon 2004:1-1452.

7. Beland FA, Mellick PW, Olson GR, Mendoza MC, Marques MM, Doerge DR: Carcinogenicity of acrylamide in $\mathrm{B} 6 \mathrm{C} 3 \mathrm{~F}(1)$ mice and $\mathrm{F} 344 / \mathrm{N}$ rats from a 2-year drinking water exposure. Food Chem Toxico/2013, 51:149-159.

8. Hogervorst JGF, van den Brandt PA, Godschalk RWL, van Schooten FJ, Schouten LJ: Interactions between dietary acrylamide intake and genes for ovarian cancer risk. Eur J Epidemiol 2017, 32:431441.

9. Wang B, Qiu W, Yang S, Cao L, Zhu C, Ma J, Li W, Zhang Z, Xu T, Wang X, et al: Acrylamide Exposure and Oxidative DNA Damage, Lipid Peroxidation, and Fasting Plasma Glucose Alteration: Association and Mediation Analyses in Chinese Urban Adults. Diabetes Care 2020, 43:1479-1486. 
10. Wang H, Feng F, Guo Y, Shuang S, Choi MMF: HPLC-UV quantitative analysis of acrylamide in baked and deep-fried Chinese foods. Journal of Food Composition and Analysis 2013, 31:7-11.

11. Pan M, Liu K, Yang J, Hong L, Xie X, Wang S: Review of Research into the Determination of Acrylamide in Foods. Foods (Basel, Switzerland) 2020, 9:524.

12. Erejuwa OO, Sulaiman SA, Ab Wahab MS: Evidence in Support of Potential Applications of Lipid Peroxidation Products in Cancer Treatment. Oxidative Medicine and Cellular Longevity 2013, 2013:931251.

13. Liu H-R, White PJ: Oxidative stability of soybean oils with altered fatty acid compositions. Journal of the American Oil Chemists' Society 1992, 69:528-532.

14. Zhang Y, Li Z, Ma Y, Mu Z: Association of total oxidant status, total antioxidant status, and malondialdehyde and catalase levels with psoriasis: a systematic review and meta-analysis. Clin Rheumatol 2019, 38:2659-2671.

15. Chen Y, Liu X, Yu Y, Yu C, Yang L, Lin Y, Xi T, Ye Z, Feng Z, Shen B: PCaLiStDB: a lifestyle database for precision prevention of prostate cancer. Database : the journal of biological databases and curation 2020, 2020:baz154.

16. Zhang FF, Cudhea F, Shan Z, Michaud DS, Imamura F, Eom H, Ruan M, Rehm CD, Liu J, Du M, et al: Preventable Cancer Burden Associated With Poor Diet in the United States. JNCl cancer spectrum 2019, 3:pkz034-pkz034.

17. Farhood B, Raei B, Malekzadeh R, Shirvani M, Najafi M, Mortezazadeh T: A review of incidence and mortality of colorectal, lung, liver, thyroid, and bladder cancers in Iran and compared to other countries. Contemporary oncology (Poznan, Poland) 2019, 23:7-15.

18. Alghamdi MA, Hassan SK, Alzahrani NA, Al Sharif MY, Khoder MI: Classroom Dust-Bound Polycyclic Aromatic Hydrocarbons in Jeddah Primary Schools, Saudi Arabia: Level, Characteristics and Health Risk Assessment. International journal of environmental research and public health 2020, 17:2779.

19. Taninaga J, Nishiyama Y, Fujibayashi K, Gunji T, Sasabe N, lijima K, Naito T: Prediction of future gastric cancer risk using a machine learning algorithm and comprehensive medical check-up data: $\mathrm{A}$ case-control study. Scientific reports 2019, 9:12384-12384.

20. Shahrbabki PE, Hajimohammadi B, Shoeibi S, Elmi M, Yousefzadeh A, Conti GO, Ferrante M, Amirahmadi M, Fakhri Y, Mousavi Khaneghah A: Probabilistic non-carcinogenic and carcinogenic risk assessments (Monte Carlo simulation method) of the measured acrylamide content in Tah-dig using QuEChERS extraction and UHPLC-MS/MS. Food Chem Toxicol 2018, 118:361-370.

21. Ahmad AS, Mayya AM: A new tool to predict lung cancer based on risk factors. Heliyon 2020, 6:e03402-e03402.

22. Bignardi C, Cavazza A, Grimaldi M, Laganà C, Manzi C, Rinaldi M, Corradini C: Acrylamide determination in baked potatoes by HPLC-MS: effect of steam and correlation with colour indices. European Food Research and Technology 2019, 245:2393-2400.

23. Tardiff R, Gargas M, Kirman C, Carson M, Sweeney L: Estimation of safe dietary intake levels of acrylamide for humans. Food and chemical toxicology : an international journal published for the 
British Industrial Biological Research Association 2009, 48:658-667.

24. Mucci LA, Wilson KM: Acrylamide intake through diet and human cancer risk. Journal of agricultural and food chemistry 2008, 56:6013-6019.

25. Raffan S, Halford NG: Acrylamide in food: Progress in and prospects for genetic and agronomic solutions. The Annals of applied biology 2019, 175:259-281.

26. Katsaiti T, Granby K: Mitigation of the processing contaminant acrylamide in bread by reducing asparagine in the bread dough. Food Addit Contam Part A Chem Anal Control Expo Risk Assess 2016, 33:1402-1410.

27. Nematollahi A, Kamankesh M, Hosseini H, Ghasemi J, Hosseini-Esfahani F, Mohammadi A, Mousavi Khaneghah A: Acrylamide content of collected food products from Tehran's market: a risk assessment study. Environmental Science and Pollution Research 2020, 27:30558-30570.

28. Asadi S, Aalami M, Shoeibi S, Kashaninejad M, Ghorbani M, Delavar M: Effects of different roasting methods on formation of acrylamide in pistachio. Food science \& nutrition 2020, 8:2875-2881.

29. Chan DS: Computer Simulation with a Temperature-Step Frying Approach to Mitigate Acrylamide Formation in French Fries. Foods 2020, 9.

30. Taubert D, Harlfinger S, Henkes L, Berkels R, Schömig E: Influence of processing parameters on acrylamide formation during frying of potatoes. J Agric Food Chem 2004, 52:2735-2739.

31. Maan AA, Anjum MA, Khan MKI, Nazir A, Saeed F, Afzaal M, Aadil RM: Acrylamide Formation and Different Mitigation Strategies during Food Processing - A Review. Food Reviews International 2020:1-18.

32. Tepper BJ, Barbarossa IT: Taste, Nutrition, and Health. Nutrients 2020, 12:155.

33. Aniołowska $\mathrm{M}$, Zahran $\mathrm{H}$, Kita $\mathrm{A}$ : The effect of pan frying on thermooxidative stability of refined rapeseed oil and professional blend. Journal of food science and technology 2016, 53:712-720.

34. Wazir H, Chay SY, Zarei M, Hussin FS, Mustapha NA, Wan Ibadullah WZ, Saari N: Effects of Storage Time and Temperature on Lipid Oxidation and Protein Co-Oxidation of Low-Moisture Shredded Meat Products. Antioxidants (Basel, Switzerland) 2019, 8:486.

35. Gao C, Sun H, Wang T, Tang M, Bohnen NI, Müller MLTM, Herman T, Giladi N, Kalinin A, Spino C, et al: Model-based and Model-free Machine Learning Techniques for Diagnostic Prediction and Classification of Clinical Outcomes in Parkinson's Disease. Scientific reports 2018, 8:7129-7129.

36. Erfani S, Ghavami M, Shoeibi S, Zand-Moghaddam A, Rastegar H: Evaluation of Fatty Acids and Volatile Compounds in Iranian Ghee by Head Space-Solid Phase Microextraction Coupled with Gas Chromatography/Mass Spectroscopy. Journal of Agricultural Science \& Technology 2020, 22.

37. Guasch-Ferré M, Liu G, Li Y, Sampson L, Manson JE, Salas-Salvadó J, Martínez-González MA, Stampfer MJ, Willett WC, Sun Q, Hu FB: Olive Oil Consumption and Cardiovascular Risk in U.S. Adults. J Am Coll Cardiol 2020, 75:1729-1739.

38. Moumtaz S, Percival BC, Parmar D, Grootveld KL, Jansson P, Grootveld M: Toxic aldehyde generation in and food uptake from culinary oils during frying practices: peroxidative resistance of a 
monounsaturate-rich algae oil. Scientific reports 2019, 9:4125-4125.

39. Geng L, Zhou W, Liu B, Wang X, Chen B: DHA induces apoptosis of human malignant breast cancer tissues by the TLR-4/PPAR-a pathways. Oncol Lett 2018, 15:2967-2977.

40. Dobaradaran S, Shabankareh Fard E, Tekle-Röttering A, Keshtkar M, Karbasdehi VN, Abtahi M, Gholamnia R, Saeedi R: Age-sex specific and cause-specific health risk and burden of disease induced by exposure to trihalomethanes (THMs) and haloacetic acids (HAAs) from drinking water: An assessment in four urban communities of Bushehr Province, Iran, 2017. Environ Res 2020, 182:109062.

\section{Supplemental Data}

Supplemental Tables A \& B are not available with this version.

\section{Figures}
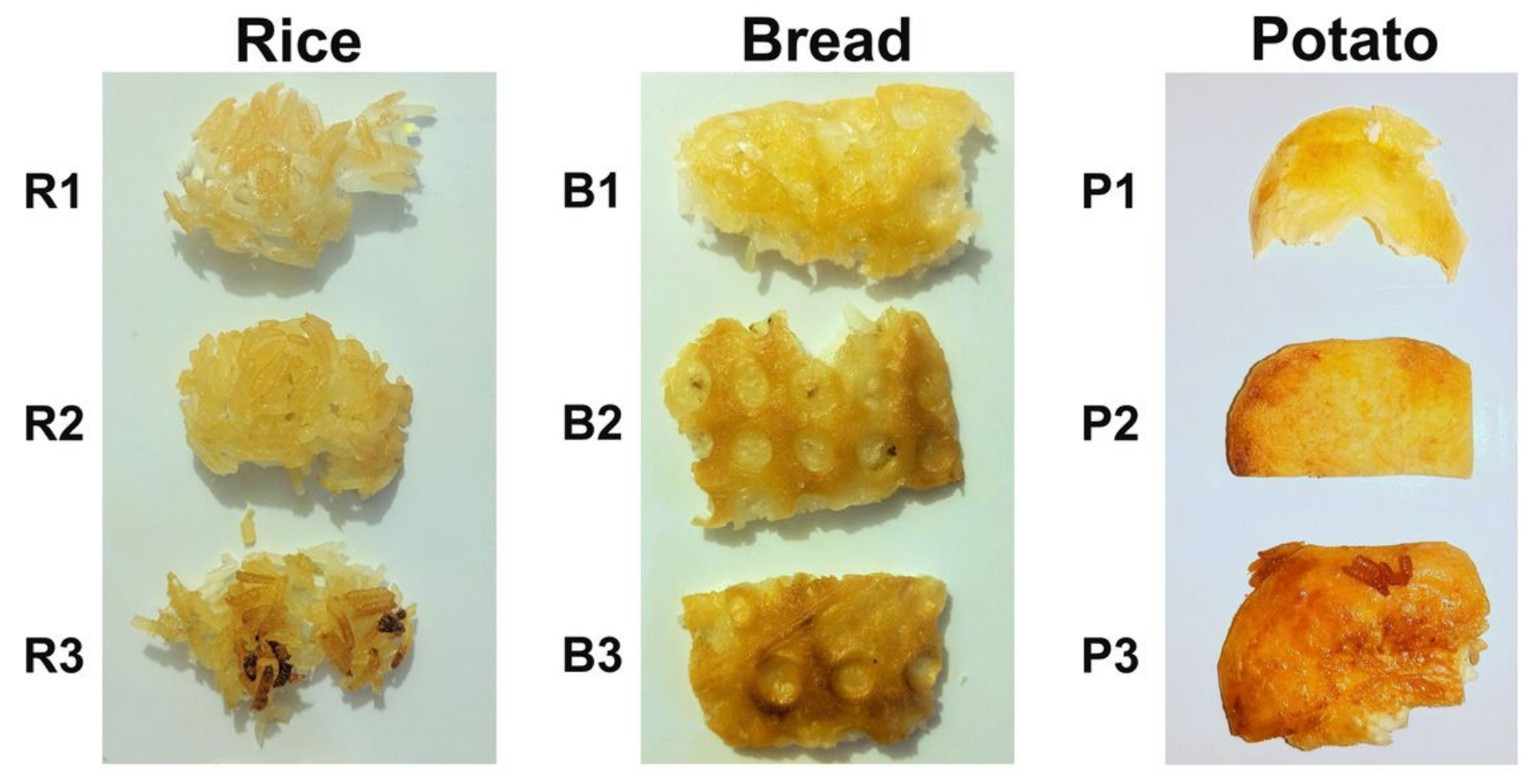

Figure 1

Tah-Dig Samples in three color ranges. Each number represents the increase in color intensity. No. $1=$ golden, No. $2=$ light brown, No. $3=$ dark brown. $\mathrm{R}=$ rice, $\mathrm{B}=$ bread, $\mathrm{P}=$ potato. Grades 1,2 , and 3 indicate an increase in color. 
A

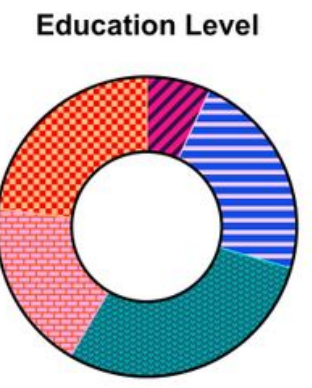

se $23.08 \%$ Postgraduate c2 $6.89 \%$ Literate

= $22.58 \%$ Elementary

$28.97 \%$ Diploma

w $18.48 \%$ Bachelor
B

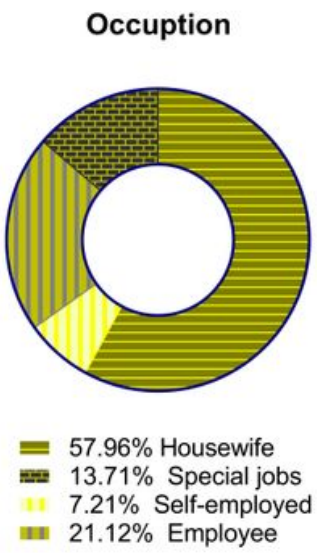

C

Frequency/week

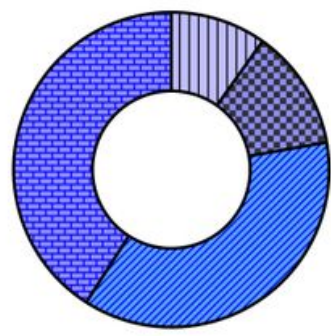

III $9.81 \%<3$ Times

as $12.41 \%$ 4-5 Times

un $36.84 \%$ 5-6 Times

40.94\% 6-7 Times
D

Types of Tah-Dig

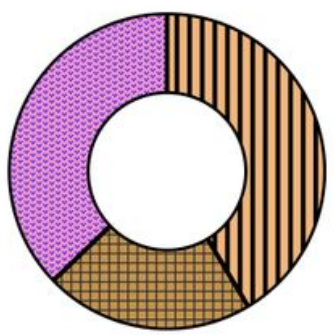

II $41.40 \%$ Bread

zys $37.20 \%$ Rice

4 III $21.40 \%$ Potato
E

\section{Cooking Time}

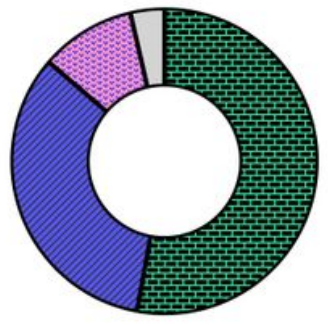

$52.90 \% \quad 15-30 \mathrm{Min}$

$10.10 \% \quad 45-60 \mathrm{Min}$

$33.40 \% \quad 30-45 \mathrm{Min}$

$=3.60 \%>60 \mathrm{Min}$

\section{Figure 2}

Data frequency distribution. (A) Frequency distribution of participants' education at five levels:

elementary, secondary, bachelor's, and postgraduate. (B) Frequency distribution related to the job of the participants in the four levels of housewife, self-employed, employee and special occupations. (C)

Frequency distribution related to the amount of consumption per week in four levels $<3,4-5,5-6,6-7$ times a week. (D) Frequency distribution related to consumption of three types of Tah-Dig bread, potatoes and rice. (E) Frequency distribution related to cooking times in four levels of 15-30, 30-45, 45-60 and> 60 minutes. 


\section{Acrylamid Content}

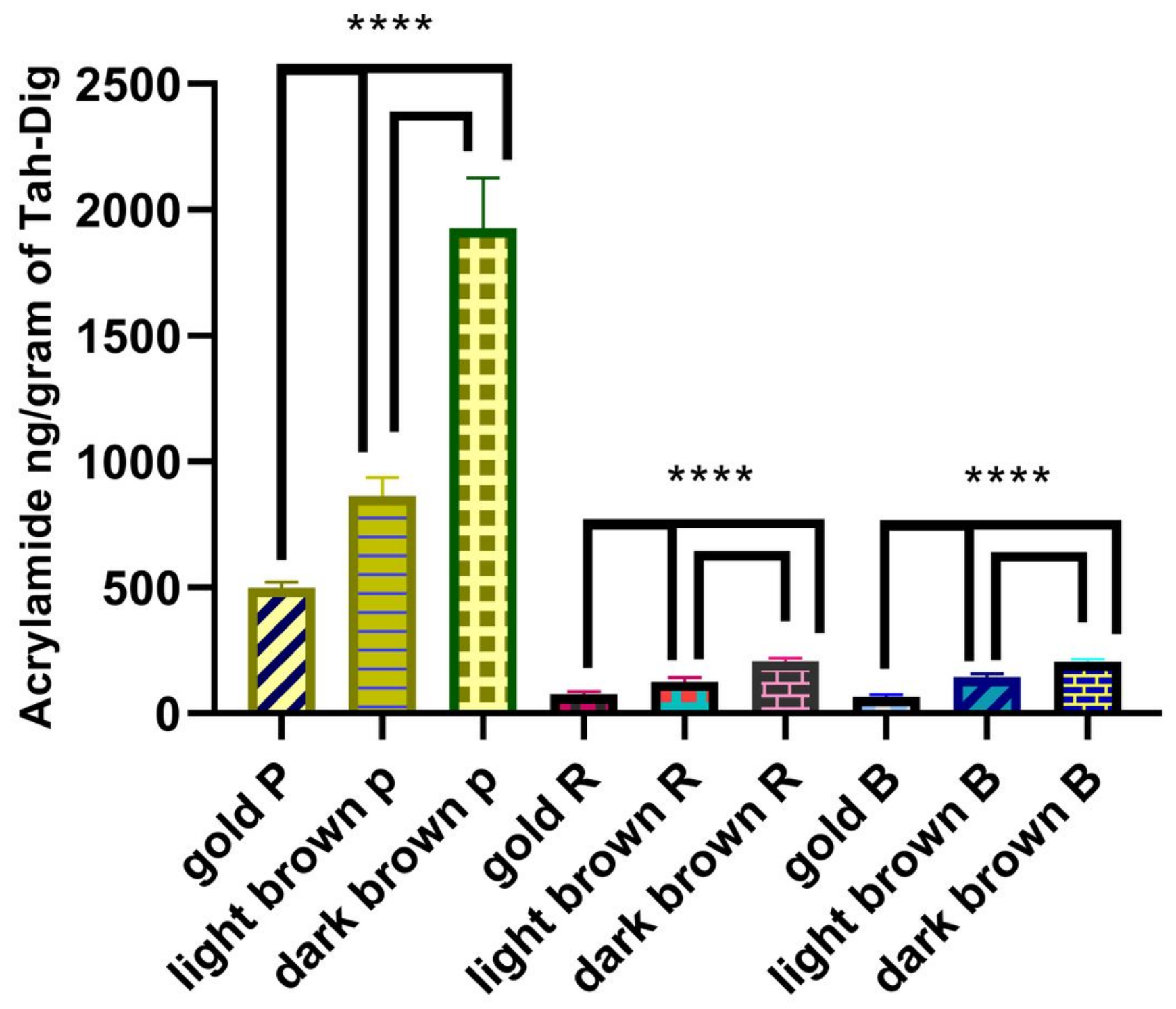

Figure 3

The amount of acrylamide in all three samples of the Tah-Dig of bread, potatoes and rice in three colors: golden, light brown and dark brown. Acrylamide levels are significantly different in intragroup and intergroup comparisons. ${ }^{\star \star \star \star}=\mathrm{P}<0.0001$. $\mathrm{B}=$ Bread, $\mathrm{P}=\mathrm{P}$ otato, $\mathrm{R}=$ Rice. 


\section{MDA (nmol/gram of Triglyceride)}

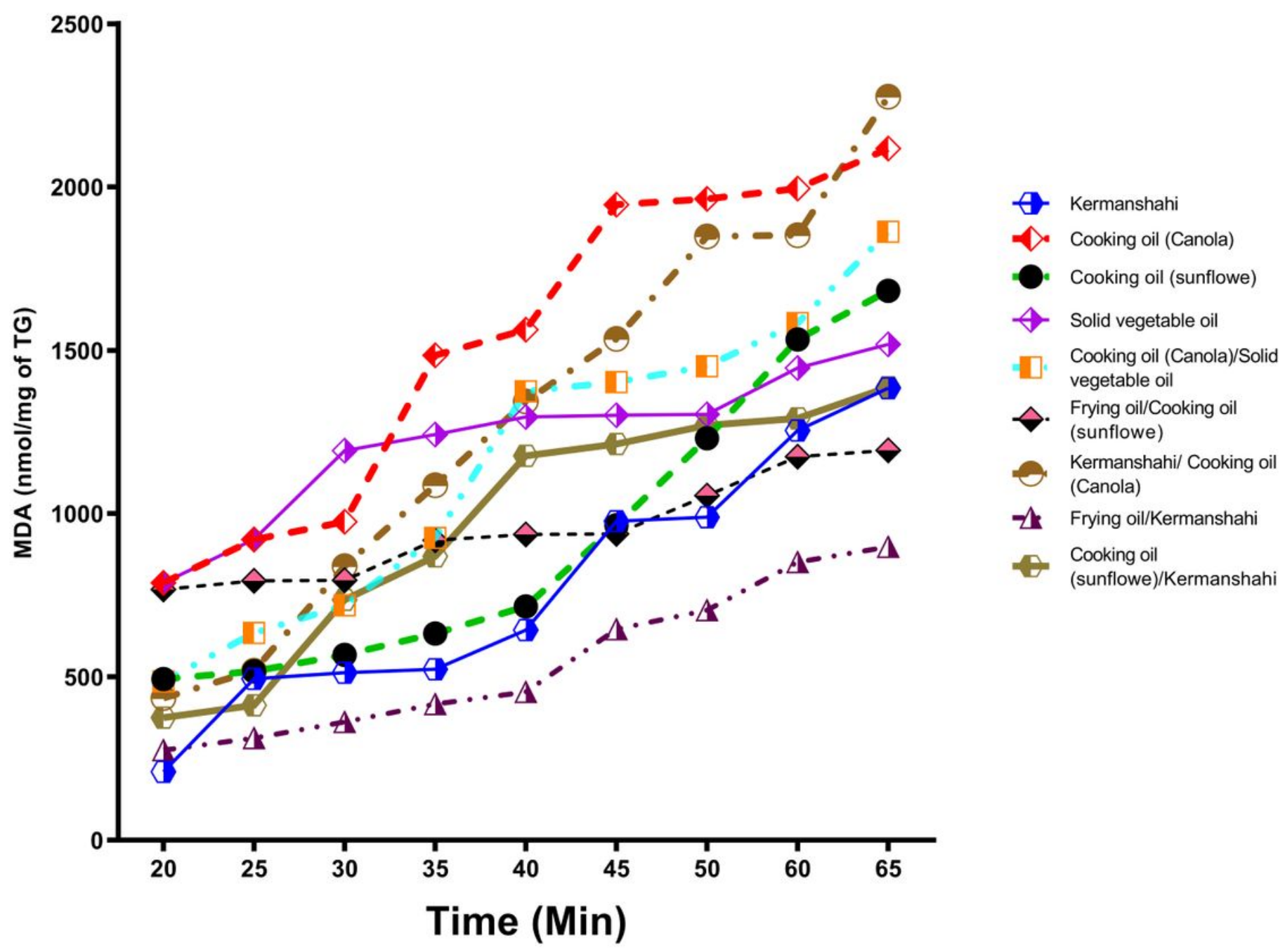

Figure 4

Malondialdehyde formation in types of oils used for Tah-Dig preparation over time. 


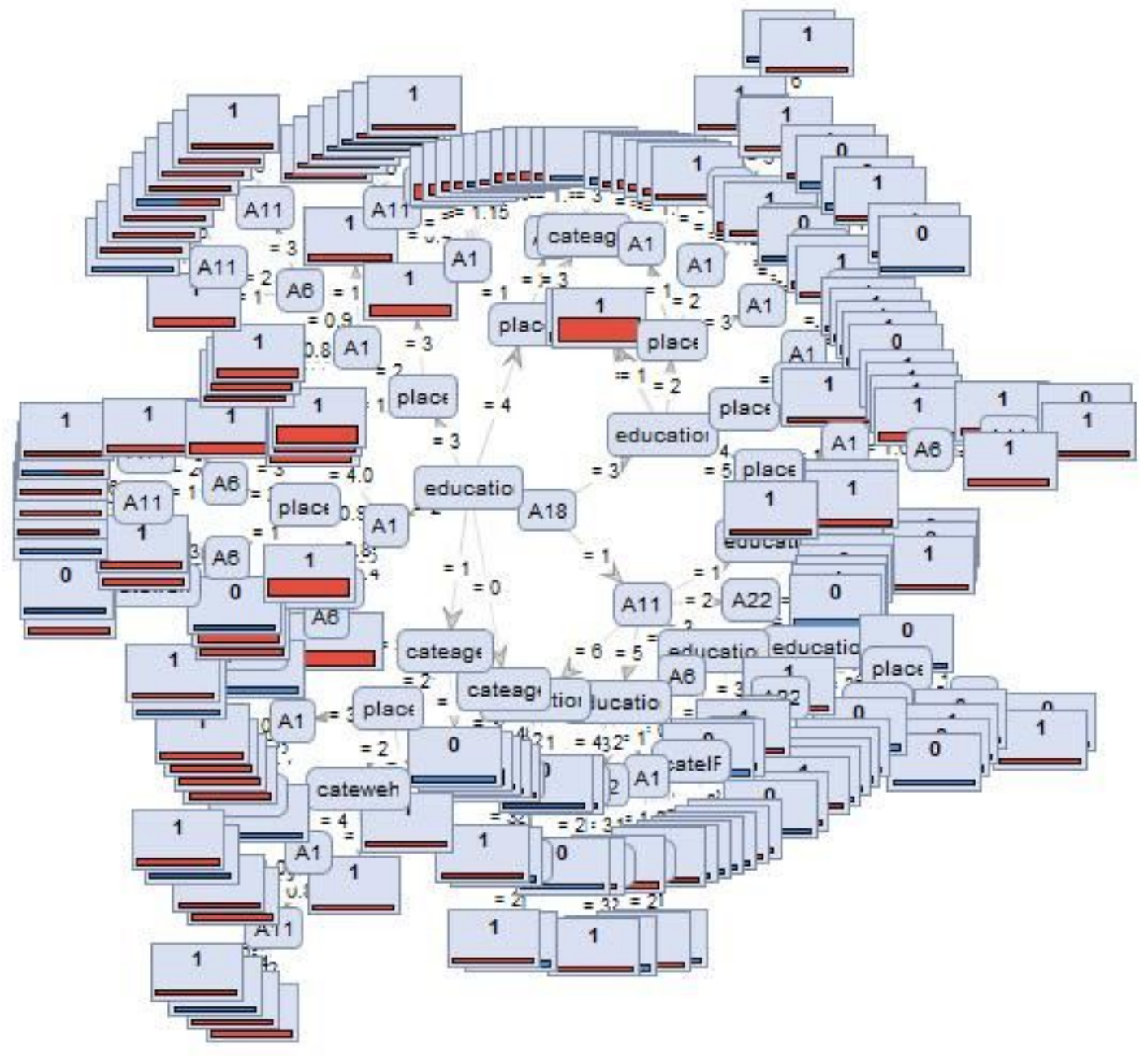

Figure 5

Decision tree. The Frequency of the features (as rules) used to predict the risk of cancer by data mining.

\section{Supplementary Files}

This is a list of supplementary files associated with this preprint. Click to download.

- GraphicalAbstract.jpg 